\title{
A Modified Image Segmentation Method Using Active Contour Model
}

\author{
Shiping Zhu ${ }^{1, a}$, Ruidong Gao ${ }^{1, b}$ \\ ${ }^{1}$ Department of Measurement Control and Information Technology, School of Instrumentation \\ Science and Opto-electronics Engineering, Beihang University, Beijing 100191, China \\ aspzhu@163.com, ${ }^{\mathrm{b}} \mathrm{rxdj1990@163.com}$
}

Keywords: Active contours; Gradient vector flow; Laplace operator; Border leakage; External force field.

\begin{abstract}
Active contours, or snakes, have extensive applications in image segmentation. Conventional snakes have several drawbacks, such as the initialization contour sensitivity and border leakage phenomenon. Many new methods have been proposed to address these problems. In this paper, we present an improved image segmentation method based on snakes. Firstly, we adopt the multi-step direction method to enlarge the scope of initial contour and obtain more precise edge map. Then, we decompose the Laplace operator to tangential direction and normal direction, weakening the border smoothing effect. Finally, two correlational self-adaptive weight functions are added to the two directions. Thus, the snakes can adaptively adjust the weights of smoothing item and diffusion item through the local image characteristics. Based on the subjective and objective evaluations, the proposed method outperforms the state-of-the-art methods and improves the segmentation accuracy.
\end{abstract}

\section{Introduction}

Active contour model, or snake model, was proposed by Kass et al [1], in 1987. Snake model has been widely applied in the fields of computer vision and image processing [2-6], such as image segmentation, target tracking, and edge detection. Although the conventional active contour model has been widely used, it still has its shortcomings [7]. First, the initial contour must be very close to the interesting image features. Second, border leakage phenomenon is occurred, losing a lot of important image information. Third, the snake curve is difficult to reach indentation boundaries.

In order to solve these problems, Xu proposed gradient vector flow (GVF) snake model [7], which is able to expand the capture scope of initial contour and has a certain indentation convergence capability. The generalized gradient vector flow (GGVF) model proposed by Xu and Prince [8] adds two weight coefficients changing in the image field based on the original GVF external force field. Thus, the curve converges rapidly in the flat field and has certain boundary protection effect. However, the indentation convergence capability has not been greatly improved. NGVF proposed by Ning [9] decomposes the Laplace operator in the GVF external force field, and only retains normal component. Thereby NGVF further improves the curve's indentation capability. Although the tangential component after decomposition has been added to GVF external force field by later NBGVF [10], the convergence capability of long and thin indentation has not been significantly improved. Based on the studies about GGVF and GVF, Qin [11] discovered that no matter GGVF or GVF can only converge to the indentation with odd pixel width and is of no convergence capability to the indentation with even pixel width. Therefore, CN-GGVF algorithm was proposed by Qin, with applying component normalization method in GGVF. CN-GGVF solved the problem that deformation curve cannot converge to even pixel width, and retained the fast convergence character in GGVF. However, CN-GGVF has no ideal effect on the protection of weak boundary features of interesting field and has boundary leakage phenomenon. 


\section{Related Work}

Traditional Snake Model. In the active contours model first proposed in [1], an energy minimizing curve (snake) is guided by external and internal energies to create a contour around an object. It can be expressed by $\mathbf{x}(s)=(x(s), y(s)), s \in[0,1]$. The energy function is as follows:

$$
E_{\text {snake }}=\int_{0}^{1}\left[E_{\text {int }}(\mathbf{x}(s))+E_{\text {ext }}(\mathbf{x}(s))\right] d s=\int_{0}^{1}\left[\alpha(s)\left|\mathbf{x}^{\prime}(s)\right|^{2}+\beta(s)\left|\mathbf{x}^{\prime \prime}(s)\right|^{2}+E_{\text {ext }}(\mathbf{x}(s))\right] d s \text {. }
$$

where $E_{\text {int }}(\mathbf{x}(s))$, the snake's internal energy or prior, is the weighted sum of first and second derivatives of $\mathbf{x}(s) . E_{\text {ext }}(\mathbf{x}(s))$ represents external energy. $\alpha(s)$ is the elastic coefficient and $\beta(s)$ denotes intensity coefficient. Based on the variational principle [12], the Eq. 1 satisfies Euler-Lagrange equation at the maximization of contour curve energy:

$$
\alpha(s) \mathbf{x}^{\prime \prime}(s)-\beta(s) \mathbf{x}^{\prime \prime \prime \prime}(s)-\nabla E_{\text {ext }}(\mathbf{x}(s))=0 .
$$

where $\mathbf{x}^{\prime \prime \prime \prime}(s)$ represents the fourth derivative of $\mathbf{x}(s)$. Eq. 2 can be regarded as force balance equation:

$$
F_{\text {int }}(\mathbf{x}(s))+F_{\text {ext }}(\mathbf{x}(s))=0 .
$$

where $F_{\text {int }}(\mathbf{x}(s))=\alpha(s) \mathbf{x}^{\prime \prime}(s)-\beta(s) \mathbf{x}^{\prime \prime \prime \prime}(s)$ represents internal forces constraining curve smoothing and stretching; $F_{\text {ext }}(\mathbf{x}(s))=-\nabla E_{\text {ext }}(\mathbf{x}(s))$ denotes external force, driving curve to move to expected feature boundaries.

GVF Snake Model. GVF snake proposed by Xu et al. will not consider snake model from the perspective of energy minimization, but consider it as a force balance process. Eq. 3 is the basic equation for the construction of GVF snake model. External force $F_{\text {ext }}(\mathbf{x}(s))$ is substituted by a GVF field $v(x, y)=[u(x, y), v(x, y)]$. The following energy function is obtained through minimize its components:

$$
E=\iint \mu\left(u_{x}^{2}+u_{y}^{2}+v_{x}^{2}+v_{y}^{2}\right)+|\nabla f|^{2}|v-\nabla f| d x d y .
$$

where $f$ represents the edge map of processed image $I ; \nabla f$ denotes gradient field of $f$; $\mu$ represents a parameter controlling the smoothness of GVF field. The gradient vector flow field satisfies the Euler equation:

$$
\left\{\begin{array}{l}
\mu \nabla^{2} u-\left(f_{x}^{2}+f_{y}^{2}\right)\left(u-f_{x}\right)=0 \\
\mu \nabla^{2} v-\left(f_{x}^{2}+f_{y}^{2}\right)\left(v-f_{x}\right)=0
\end{array} .\right.
$$

where $f_{x}=\frac{\partial f}{\partial x}, f_{y}=\frac{\partial f}{\partial y}$. The first term is diffusion item, and $\nabla^{2}$ is Laplace operator.

\section{The Proposed Method}

Multi-step Directional Approach. In GVF snake model, boundary image is gained through the image gradient and external force field is achieved based on the boundary information. It is very 
necessary to get an accurate boundary image. However, GVF snake model only retains the amplitude value of gradient image, losing gradient direction information. With a complicated background, snake curve may easily converge to false boundaries. For driving snake curve to converge more correctly to the desired boundary, we optimize the process of capturing a boundary image. The flow chart is shown in Fig. 1. The brief steps are as follows:

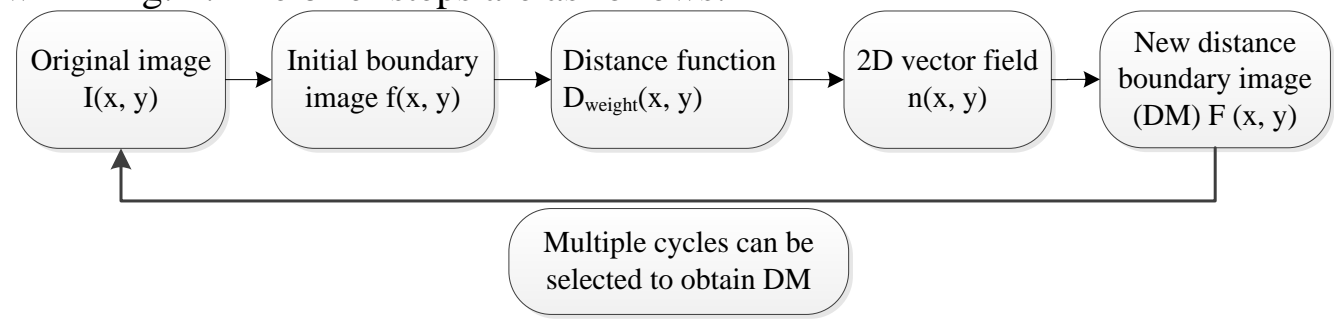

Fig. 1 The flow chart of multi-step directional approach

Step 1: Use (6) to obtain initial boundary image $f(x, y)$ :

$$
f(x, y)=\nabla\left(\mathrm{G}_{\sigma}(x, y) \times I(x, y)\right) .
$$

Step 2: Add gradient direction information to obtain a new DM (Distance Map), denoted by $D_{w}(x, y)$ [13]. Consider DM as the final boundary image to apply it in the iterative process.

Step 3: Define 2D vector field $n(x, y)$ depending on DM function:

$$
n(x, y)=\left\{\begin{array}{ll}
\nabla D_{w}(x, y) & \text { if }(\mathrm{x}, \mathrm{y}) \text { is inside the contour } \\
\nabla \varphi(x, y) & \text { if }(\mathrm{x}, \mathrm{y}) \text { is on the contour } \\
-\nabla D_{w}(x, y) & \text { if }(\mathrm{x}, \mathrm{y}) \text { is outside the contour }
\end{array} .\right.
$$

where $\varphi(x, y)$ can be defined voluntarily or use a function in the known methods. We use the same $\varphi(x, y)$ with [13]. $n(x, y)$ has unit amplitude and ideal directivity.

Step 4: The new distance boundary image can be gained by convoluting $n(x, y)$ and $f(x, y)$ :

$$
f_{\text {new }}(x, y)=\left\{\begin{array}{ll}
f(x, y) \cdot n(x, y) & f(x, y) \cdot n(x, y) \geq 0 \\
0 & f(x, y) \cdot n(x, y)<0
\end{array} .\right.
$$

Step 5: Select multiple cycles to gain boundary image depending on the complexity of image background. Generally, the cycle just needs to be performed once.

Adaptive Diffusion. Laplace operator is used as diffusion item in GVF snake model and it is shown below:

$$
\nabla^{2}=\frac{\partial^{2}}{\partial x^{2}}+\frac{\partial^{2}}{\partial y^{2}}
$$

In Eq. 5, $\nabla^{2} u$ and $\nabla^{2} v$ are diffusion items, $\left(u-f_{x}\right)$ and $\left(v-f_{y}\right)$ are data items. The diffusion items have strong anisotropic properties, but do not have the capability in protecting boundaries. Nevertheless, data items can protect the boundaries. Therefore, in order to improve boundary protection of snake curve, we decomposes Laplace operator in the tangential and normal directions. 


$$
\left\{\begin{array}{l}
\nabla^{2} L=L_{T T}+L_{N N} \\
L_{T T}=\frac{1}{|\nabla L|^{2}}\left(L_{x}^{2} L_{y y}+L_{y}^{2} L_{x x}-2 L_{x} L_{y} L_{x y}\right) \\
L_{N N}=\frac{1}{|\nabla L|^{2}}\left(L_{x}^{2} L_{x x}+L_{y}^{2} L_{y y}+2 L_{x} L_{y} L_{x y}\right)
\end{array}\right.
$$

where $L_{T T}$ and $L_{N N}$ represent the tangential and normal second derivatives of $L$ respectively. So Eq. 5 can be rewritten as:

$$
\left\{\begin{array}{l}
u=g(|\nabla f|) \cdot\left(l(|\nabla f|) u_{T T}+s(|\nabla f|) u_{N N}\right)-h(|\nabla f|)\left(u-f_{x}\right) \\
v=g(|\nabla f|) \cdot\left(l(|\nabla f|) v_{T T}+s(|\nabla f|) v_{N N}\right)-h(|\nabla f|)\left(v-f_{y}\right)
\end{array} .\right.
$$

where correlational self-adaptive weighting functions $l|\nabla f|$ and $s|\nabla f|$ are defined as follows:

$$
\left\{\begin{array}{l}
l|\nabla f|=1-e^{-\frac{|\nabla f|^{2}}{R^{2}}} \\
s|\nabla f|=e^{-\frac{|\nabla f|^{2}}{R^{2}}}
\end{array} .\right.
$$

Parameter $R$ is a positive constant. If the boundaries are weaker, the value of $R$ is relatively smaller. On the contrary, the value of $R$ is higher, which can be appropriately adjusted based on different features of image boundaries. Divergence along the tangential direction has the function to protect image boundaries, whereas, the diffusion along the normal direction functions to smooth noise and image boundaries.

\section{Experimental Results}

In order to verify the effect of proposed snake model, we carried out experiments on synthetic image and real image. We compared with the existing GVF [7], GGVF [8], NGVF [9], NBGVF [10], CN-GGVF [11], and analyzed experimental results in turn. All the experiments selected C++ language as the implementation language. Visual Studio 2008 is used as the development environment. CPU with Intel ${ }^{\circledR}$ Core ${ }^{\mathrm{TM}}$ i7-3770, 3.40GHz and $4 \mathrm{G}$ memory size.

Synthetic Image. Since Kass proposed snake model in 1987, the indentation convergence capability of snake curve has become an important standard to evaluate a new snake model. Although several current typical snake models have made many improvements, it still has large space to make efforts to the ability of long and thin indentation (LTI). The green curve in Fig. 2 (a) represents the initial contour of snake, which is generated automatically. As shown in Fig. 2, GVF, GGVF, NGVF, and NBGVF cannot converge to the bottom of LTI effectively. However, the proposed method can converge to the bottom of LTI and has better corner convergence and protection effect compared with CN-GGVF, further improving LTI convergence capability of snake curve. ( $\alpha=0.8, \beta=0.1$, number of iterations is $40, R=0.8$ ) 


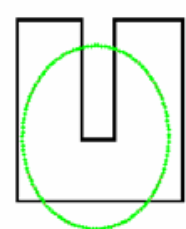

(a)

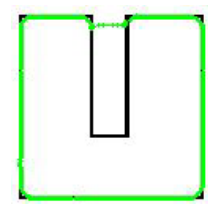

(b)

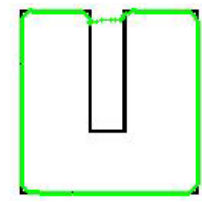

(c)

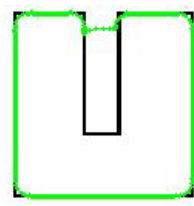

(d)

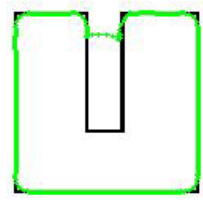

(e)

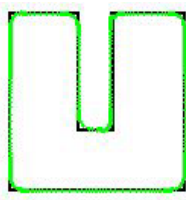

(f)

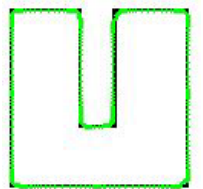

(g)

Fig. 2 Segmentation results of long and thin indentation using differnet snake models. (a) Initial contour (b) GVF (c) GGVF (d) NGVF (e) NBGVF (f) CN-GGVF (g) Proposed method.

Real Image. We take live-action image "Standing" to test the algorithm, as shown in Fig. 3. In Fig. 3 (a), the red line with green control point is the initial contour, which is given manually on the gray image. From Fig. 3 (b) to Fig. 3 (e), we display the segmentation results of color image. As we can see, the crack in the wall is the boundary in background. Snake curves in Fig. 3 (b) and Fig. 3 (d) converge to the crack, causing incorrect segmentation results. In Fig. 3 (c), the curve comes across the boundary of right hand and converges to the collar, losing foreground information. However, the proposed method, shown in Fig. 3 (e), has the correct segmentation results and eliminates the boundary leakage problem. ( $\alpha=0.6, \beta=0.1$, number of iterations is $60, R=0.5$ )

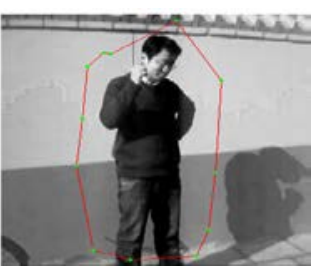

(a)

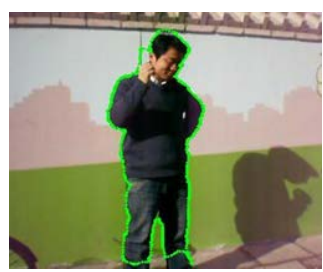

(b)

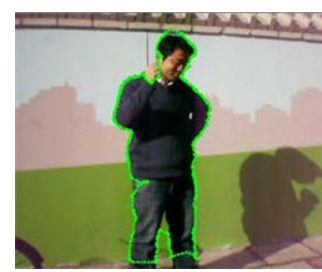

(c)

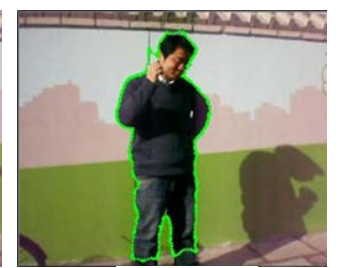

(d)

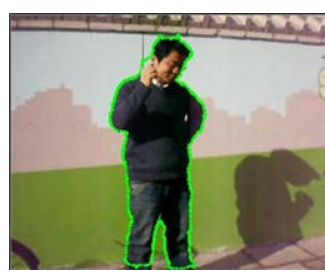

(e)

Fig. 3 Segmentation results of real image "Standing" using differnet snake models. (a) Initial contour (b) GGVF (c) NBGVF (d) CN-GGVF (e) Proposed method.

The subjective evaluation about algorithm performance has some limitations. Precision, recall and F1 measure are adopted to evaluate the segmentation results objectively [14]. The ground truth is obtained manually by using Photoshop CS5. The comparison histogram is shown in Fig. 4. The detailed data are given in Table 1. Obviously, the proposed snake model has more precise segmentation results than GGVF, NBGVF and CN-GGVF.

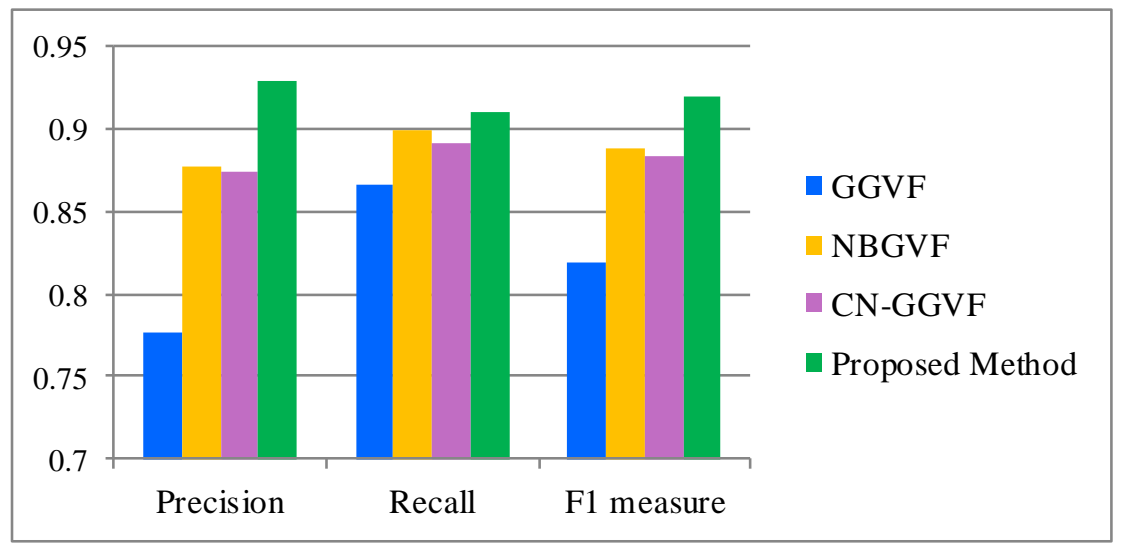

Fig. 4 Histogram of objective evaluation on real image "Standing" using precision, recall and F1 measure.

Table 1 Objective evaluation results on real image "Standing" using precision, recall and F1 measure.

\begin{tabular}{|c|c|c|c|}
\hline Methods Criteria & Precision & Recall & F1 measure \\
\hline GVF & 0.7765 & 0.8666 & 0.8191 \\
\hline NBGVF & 0.8764 & 0.8991 & 0.8876 \\
\hline CN-GGVF & 0.8739 & 0.8920 & 0.8829 \\
\hline Proposed Method & 0.9283 & 0.9104 & 0.9193 \\
\hline
\end{tabular}




\section{Summary}

Conventional snake models have several drawbacks in the field of image segmentation. In order to obtain more precise results, we apply multi-step directional approach and add directional information of vector field to acquire boundary image. In the meantime, we add tangential and normal correlational self-adaptive weighting functions after Laplace operator decomposition. Compared with other snake models, the experimental results show that the proposed snake model has better convergence ability and image segmentation effect.

\section{Acknowledgment}

This research is funded by the National Natural Science Foundation of China (NSFC) under grants No. 61375025, No. 61075011, and No. 60675018, also the Scientific Research Foundation for the Returned Overseas Chinese Scholars from the State Education Ministry of China.

\section{References}

[1] M. Kass, A. Witkin and D. Terzopoulos: Snakes: Active contour models. International Journal of Computer Vision, Vol. 1, no. 4 (1988), pp. 321-331.

[2] C.Davatzikos, J. Prince: An active contour model for mapping the cortex. IEEE Transactions on Medical Imaging Processing, Vol. 1, no. 14 (1995), pp. 65-80.

[3] J. Tang, S. Millington, S. Acton, J. Crandall and S. Hurwitz: Surface extraction and thickness measurement of the articular cartilage from MR images using directional gradient vector flow snakes. IEEE Transactions on Biomedical Engineering, Vol. 53, no. 5 (2006), pp. 896-907.

[4] J. Tang: A multi-direction GVF snake for the segmentation of skin cancer images. Pattern Recognition, Vol. 6, no. 42 (2009), pp. 1172-1179.

[5] F. Zhang, X. Zhang and K. Cao: Contour extraction of gait recognition based on improved GVF Snake model. Computers and Electrical Engineering, Vol. 4, no. 38 (2012), pp. 882-890.

[6] Q. Li, Y. Wang and H. Liu: A combined spatial-spectral method for automated white blood cells segmentation. Optics and Laser Technology, Vol. 30, no. 54 (2013), pp. 225-231.

[7] C. Xu, J. Prince: Gradient vector flow: a new external force for snakes. IEEE Conference on Computer Vision and Pattern Recognition (CVPR’97), Vol. 17-19 (1997), pp. 66-71.

[8] C. Xu, J. Prince: Generalized gradient vector flow external forces for active contours. Signal Processing, Vol. 2, no. 71 (1988), pp. 131-139.

[9] J. Ning, C. Wu and S. Liu: NGVF: An improved external force field for active contour model. Pattern Recognition Letters, Vol. 1, no. 28 (2007), pp. 58-63.

[10] Y. Wang, L. Liu, H. Zhang, Z. Cao and S. Lu: Image Segmentation Using Active Contours With Normally Biased GVF External Force. IEEE Signal Processing Letters, Vol. 17, no. 10 (2010), pp. 875-878.

[11]L. Qin, C. Zhu, Y. Zhao, H. Bai and H. Tian: Generalized Gradient Vector Flow for Snakes: New Observations, Analysis, and Improvement. IEEE Transactions on Circuits and Systems for Video Technology, Vol. 23, no. 5 (2013), pp. 883-897.

[12]G. Aubert, P. Kornprobst, Mathematical problems in image processing: partial differential equations and the calculus of variations (Springer Publications, Germany 2006), pp. 123-135.

[13] Y. Le, X. Xu and Z. Li: A multi-step directional generalized gradient vector flow snake for target tumor segmentation in US-guided high-intensity focused ultrasound ablation. Biomedical Signal Processing and Control, Vol. 6, no. 8 (2013), pp. 81-821. 
[14] D. Lui, C. Scharfenberger, K. Fergani, A. Wong, and D. A. Clausi: Enhanced Decoupled Active Contour Using Structural and Textural Variation Energy Functionals. IEEE Transactions on Image Processing, Vol. 23, no. 2 (2014), pp. 855 - 869. 